\title{
Perspectivas teóricas de Cibercultura, y su aplicación en espacios educativos
}

Theoretical perspectives of Cyberculture, and its application to educational spaces.

Jossue Cabrera García

Departamento de Antropología Social, UCM. Madrid (España)

jcabrera@ucm.es

\begin{abstract}
RESUMEN
Las nuevas tecnologías están presentes en la vida cotidiana de las personas en la sociedad de la información. Esta cotidianidad ha interesado a científicos sociales, realizando numerosos estudios en y de contextos en los que se integran los dispositivos tecnológicos, siendo más frecuente el estudio en la escuela o la universidad que en otros espacios educativos, debido, entre otros factores, al especial interés sobre la juventud y la infancia que tiene que capacitarse tecnológicamente para la ciudadanía del siglo XXI. Así, el presente estado de la cuestión trata recoger trabajos teóricos y prácticos relacionados con las nuevas tecnologías desde dos áreas de investigación, el antropológico y el educativo.
\end{abstract}

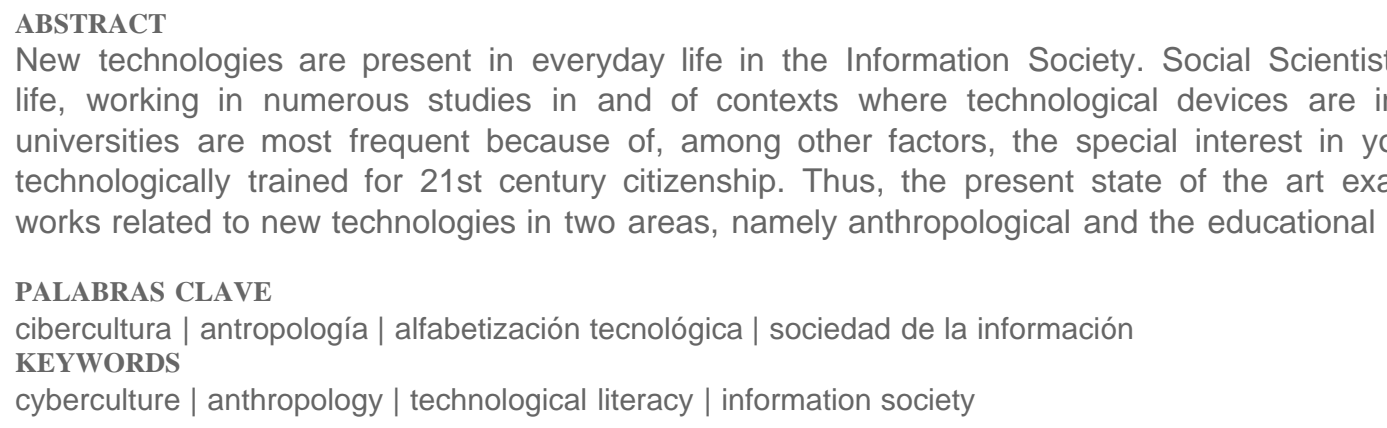
works related to new technologies in two areas, namely anthropological and the educational

PALABRAS CLAVE

cibercultura | antropología | alfabetización tecnológica | sociedad de la información KEYWORDS

cyberculture | anthropology | technological literacy | information society life, working in numerous studies in and of contexts where technological devices are involved. Studies in schools or the universities are most frequent because of, among other factors, the special interest in youth and children, who need to be technologically trained for 21st century citizenship. Thus, the present state of the art examines some theories and practical

\section{Introducción}

Los centros educativos han introducido de forma paulatina las nuevas tecnologías en el ámbito de la educación formal y la no formal en los últimos quince años, buscando el aprendizaje de ciertas capacidades que se necesitan en la llamada sociedad de la información. Para entender esto es necesario, por un lado comprender cuáles son estas nuevas capacidades y qué las diferencian de las no nuevas y, por otro lado, qué es la sociedad de la información.

El concepto sociedad de la información ha sido discutido ampliamente por Castells en sus libros La era de la información: economía, sociedad y cultura y La sociedad red: una visión global. Aunque inicialmente el concepto se asoció a la globalización de la economía, pronto comenzó a utilizarse para referirse a otros aspectos de la cotidianidad humana acontecidos con la expansión de las tecnologías de la información y la comunicación (TIC) en la vida diaria de los agentes sociales.

Proporcionar la capacidad de acceder a estos flujos de información será el objetivo principal de la alfabetización tecnológica en la era digital. Para ello, la alfabetización tecnológica tratará de capacitar a las personas para acceder a las redes virtuales de comunicación e información. Entre estas nuevas capacidades se encuentran la compresión del lenguaje audiovisual, el hipertextual y el multimedia, la capacidad de navegar por Internet, de buscar información e interpretarla de forma crítica y de comunicarse utilizando los dispositivos tecnológicos y configurando éstos para sus propios usos, es decir, convertirse en un agente social activo. 
La alfabetización tecnológica o digital es una propuesta hecha por investigadores de la educación que responde a nuevas necesidades educativas, orientadas a que las personas puedan intervenir en el ámbito personal, laboral y público (en relación a ciudadanía) en la sociedad de la información (Cope y Kalantzis 2000). Sin embargo, estas propuestas educativas no están llegando a todos por igual. Muchos autores coinciden en el aumento de la división o brecha digital, o tecnológica (Bautista García-Vera 2007, Castells 2004, Cope y Kalantzis 2009, Gros y Contreras 2006, Martínez Sánchez 2009, Piscitelli 2002, Prensky 2001) entre aquellos que tienen acceso a las nuevas tecnologías y aquellos que no, ahora incrementada por quienes tienen acceso a la formación para usarlas y quiénes no.

De cualquier manera, aun teniendo acceso a las nuevas tecnologías y recibiendo formación sobre éstas, no se asegura la disminución de la llamada brecha tecnológica. Uno de los conceptos que se han desarrollado para explicarlo es el de inmigrante digital, categoría que ha sido discutida principalmente por Marc Prensky y Alejandro Piscitelli. Ésta ha venido utilizándose para referirse a las personas que han crecido con un tipo de lenguaje analógico y de descodificación de significados textuales u orales y tienen que adaptarse a las nuevas circunstancias de la sociedad informacional, con un tipo de lenguaje digital y a la descodificación de significados hipertextuales. Estos autores, además, predicen que estas diferencias entre los que han crecido con las nuevas tecnologías, nativos digitales, y aquellos que han adquirido más tarde los procesos cognitivos para usarlas conllevarán problemas comunicativos y de desigualdades de acceso a los beneficios online de participación pública, económica, cultural etc.

Con esta introducción se ha tratado de sacar a la luz algunos conceptos importantes para entender la elección de los textos que se desarrollan a continuación, cuyos intereses son, por un lado, recoger algunas líneas teóricas antropológicas en relación a la comunicación mediada por dispositivos tecnológicos; y por otro, ir viendo qué tipo de trabajos se han desarrollado desde la educación, extrayendo el modelo teórico de cibercultura utilizado.

\section{Antropología y educación, y su relación con las tecnologías de la información y la comunicación}

\subsection{Antropología y TIC}

El término ciber ha estado asociado a la tecnología informática y más tarde a los medios de comunicación. En el ámbito antropológico la cibercultura puede hacer referencia a todos los cambios de índole cultural que se están generando como consecuencia de la utilización de la informática y las telecomunicaciones, aunque esta concepción o posición teórica no es la única, sino que responde a un modo concreto de entender la cultura. Este trabajo reflexiona sobre el concepto y las diferentes posiciones que se han tomado al respecto.

Para el análisis de las diferentes posturas sobre las nuevas tecnologías, voy a utilizar una clasificación propuesta por Elisenda Ardèvol. Esta autora intenta, por un lado, entender qué significa cibercultura y su relación con el concepto general de cultura. Y, por otro, discernir cuáles son los usos dados a la cibercultura. Distingue una definición ancha del concepto de una definición estrecha, siendo esta última una acepción que centra el estudio de la cibercultura en la actividad en línea o en los mitos generados en torno a Internet (Ardèvol 2003: 3). La definición ancha se refiriere a la aparición de un nuevo orden cultural, precisamente el de la cibercultura.

\subsubsection{Cibercultura como modelo cultural}

Estrategia de investigación orientada al análisis del cambio social estructural, con énfasis en los aspectos evolutivos y adaptativos para detectar la transformación cultural. El concepto de cultura que está involucrado en ella apunta a que los cambios se producen en el sistema cultural entendido en su totalidad, y hace referencia a la relación entre desarrollo tecnológico y cambio social, determinismo tecnocientífico y tecnoeconómico.

Este determinismo tecnológico se interesa por el impacto de las nuevas tecnologías, como Internet, 
sobre los agentes sociales (Negroponte 1995), un impacto tenido por incuestionable e inevitable, por lo que sólo queda adaptarse a ellos. Perspectiva que podemos encontrar en los estudios relacionados con las nuevas tecnologías en los espacios educativos.

Pierre Lévy (1999a, 1999b, 2001) pese a no aceptar que la causa del desarrollo cultural sea la tecnología o que ésta sea independiente de factores sociales, sitúa Internet en el centro de una transformación cultural singular y sin precedentes, hablándonos de una mutación. Define tres principios claves de la cibercultura: la interconectividad, las comunidades virtuales y la inteligencia colectiva. En su opinión, esta cibercultura corresponde a la etapa de la mundialización concreta de las sociedades, de la coexistencia entre los niveles locales y globales.

La denominada Escuela de Toronto, siguiendo los estudios de Marchall Mc Luhan, se plantea los efectos psicológicos y sociales de las TIC, y propone el concepto de psicotecnología para definir cualquier tecnología que extienda, imite o amplíe los poderes de nuestras mentes. Explica la sociedad actual a partir del impacto de las nuevas tecnologías, y la inteligencia colectiva como los cambios cognitivos de la persona misma en la "aldea global" (De Kerckhove 1999: 33).

Las TIC como modelo cultural no sólo tienen una importante influencia en nuestra vida cotidiana, sino también en las representaciones del mundo, en la configuración de éste y en las expectativas y exigencias que asumimos. Así, para Piscitelli (2002), la cibercultura se enmarca en la relación entre los humanos y las máquinas, por lo que hay que explorar las transformaciones multinivel que conlleva el desarrollo tecnológico. Para comprender esta relación hombre/máquina, este autor propone una nueva área de investigación: la etnotecnología. Piscitelli, en la primera parte de su libro Ciberculturas 2.0: en la era de las máquinas inteligentes, desarrolla la relación que mantienen humanos y maquinas; la segunda parte trata sobre mundos virtuales; y la tercera parte sobre Internet, haciéndose la pregunta de si es una revolución o una evolución. La dialéctica entre la cultura y la tecnología le sirve de marco para el análisis de lo que llama "metamorfosis cognitiva" (Piscitelli 2002: 19), poniendo múltiples ejemplos de ésta. Uno de ellos es el paso de la escritura al texto electrónico, un tránsito del modo de lectura, del texto al hipertexto, que entraña diferentes modos de inteligibilidad.

Este autor habla de "planos evolutivos" (Piscitelli 2002: 34) para referirse a los continuos cambios de paradigmas que la entrada de contribuciones científicas y tecnológicas ha favorecido, y habla de una paulatina descentralización del yo en la era actual, a la que denomina era poshumana por la introducción de la inteligencia artificial (IA). Estos cambios de paradigmas han ido de la mano del aumento de la inteligencia a través del flujo de las nuevas tecnologías y del mutualismo hombre-máquina. Piscitelli plantea una metamorfosis de la virtualidad que comienza con las comunidades textuales, continúa con el auge de las tecnologías audiovisuales, después con las comunidades electrónicas y de entretenimiento, le sigue las comunidades informáticas, y finalmente las comunidades virtuales y ciberespaciales en las que estaríamos en la actualidad.

Para Piscitelli, la innovación tecnológica es como el nacimiento de una nueva especie biológica, ya que para él la tecnología son "nuevas prácticas y herramientas para conducir las organizaciones y la vida humana (...), donde diseñar nuevas herramientas es diseñar nuevas prácticas" (Piscitelli 2002: 62). En todo caso, el flujo tecnológico, afirma Piscitelli, se consolida en un proceso de interminable hibridación entre tecnologías.

La comunidad virtual dispone de un nuevo espacio donde interactuar promovido por las nuevas tecnologías: el ciberespacio, referido al espacio en red de los actores conectados, que configuran y estabilizan redes de relaciones sociales que modifican los procesos cognitivos, la interacción social y, por consiguiente, la sociedad y la cultura. En el ciberespacio, la interacción cara a cara desaparece por la intermediación de las máquinas y el lugar físico y el tiempo se transforman, lo que conlleva también una transformación cognitiva. Para comprender este nuevo campo es necesaria, según el mismo autor, una nueva metodología: la ya mencionada, etnotecnología.

Desde esta perspectiva, podríamos hablar de personas que ya están incorporadas en esta cibercultura, que han crecido con las nuevas tecnologías, y de otras que aún siguen en estadios anteriores y, por tanto necesitan aprender a ser ciber-ciudadanos. Algunos autores distinguen a unas de otras mediante la 
dicotomía nativos digitales e inmigrantes digitales. En el año 2001, Marc Prensky introdujo estos conceptos, que desarrolló en dos de sus artículos: "Digital Natives, Digital Immigrants" y "Do They Really Think Differently?" . En el primero de ellos, muestra el deseo de abrir el debate acerca de una nueva perspectiva que ayude a la docencia en el escenario digital. En la exposición de su argumento, Prensky describe las diferencias y la discontinuidad que existe entre los jóvenes que han nacido y crecido con las tecnologías digitales, a los que llama nativos digitales, y los que han incorporado esta tecnología más tarde en sus vidas, esto es, los inmigrantes digitales. Éstos últimos han tenido que adaptarse a los ambientes y a las situaciones particulares, pero tienen algo común y es un marcado "acento del pasado" (Prensky 2001a: 6). Para Prensky esta brecha tiene consecuencias sobre la educación, pues -según sostiene- los profesores, como inmigrantes digitales, son incapaces de instruir a unos alumnos nativos, que ven en el profesor a un extranjero que apenas es capaz de comunicarse.

Años más tarde, en 2004, Marc Prensky publica "¿La muerte del mando y el control?", texto en el que sigue exponiendo su argumento, a saber: que los cambios sin precedentes en tecnologías que hemos tenido en los últimos treinta años han configurado nuevos patrones de pensamiento, especialmente en los jóvenes, los mayores usuarios de las mismas; que a su vez estos cambios han permitido transformaciones en el comportamiento, algunas de las cuales han sido estudiadas parcialmente, pero rara vez en su totalidad.

El ya citado Alejandro Piscitelli (2006), en la línea de Prensky, conceptualiza a los inmigrantes digitales retomando las categorías pre y post figurativas de Margaret Mead. Al contrario que Prensky, que identifica la brecha generacional con la brecha digital, Piscitelli las diferencia. La brecha digital afecta al modo de adquisición de valores, conceptos, visiones del mundo, habilidades y competencias tecnológicas diferentes entre nativos e inmigrantes, pero se puede dar entre personas de la misma generación y tiene más que ver con el "estatus socioeconómico" (Piscitelli 2006: 179). Así, la brecha generacional es el distanciamiento en las formas de acceder a las nuevas tecnologías teniendo en cuenta la variable edad, pues las personas jóvenes se adaptan mejor a ellas. Para este autor, las nuevas tecnologías son el puente hacia el mundo cultural y cognitivo digital, así el que las personas jóvenes se adapten mejor a las nuevas tecnologías disminuirá el riesgo que la brecha digital supone, riesgo a la exclusión social en el contexto de la sociedad de la información (Castells 2004), por lo que la brecha generacional influiría en la brecha digital.

Juan de Pablos Pons (2008) advierte del problema y del reto que la brecha digital puede suponer para la sociedad informacional: mayores desigualdades entre las personas que no tienen acceso y aquellas otras con acceso a los flujos de información, culturales, sociales y económicos a través de las nuevas tecnologías. Este acceso no es sólo disponer de los dispositivos tecnológicos sino también de la comprensión del fenómeno mediático en su conjunto, entender las posibilidades que ofrecen de forma integral las TIC para poder tener una visión crítica. Por ello, De Pablos insiste en los esfuerzos por disminuir esta brecha digital empezando por la introducción de las nuevas tecnologías en los proyectos educativos de las escuelas.

Para los autores que entienden la cibercultura como un nuevo modelo cultural, las TIC han revolucionado la economía, los sistemas de comunicación, las referencias temporales de nuestras actividades e incluso las formas de hacer política a escala global.

\subsubsection{Cibercultura como formas culturales}

Siguiendo con el esquema de Ardévol (2003), cibercultura como forma cultural abarca el estudio de los aspectos culturales vinculados a la interacción social mediada por ordenador (CMO). La CMO constituye una cultura específica con características propias y diferenciadas de la realidad offline. Cibercultura, en este sentido, hace referencia a los rasgos culturales que caracterizan a las comunidades virtuales y que emergen a través de la interacción en línea. Ello supone que las interacciones que se dan mediadas por las tecnologías en red configuran nuevas formas de pensar, de actuar, de relacionarse, propias de una comunidad online. Así, la investigación está dirigida a comprobar que la vida social online tiene los mismos atributos que cualquier sistema cultural y a definirlos. Para conocer estos rasgos del sistema cultural online, esta perspectiva utiliza la "etnografía virtual" (Domínguez Figaredo et al. 2007), con técnicas como la observación participante online y la entrevista online. 
Uno de los autores más representativos de este posicionamiento es Howard Rheingold quien asegura que el futuro de la cibercultura pasa por lo bien que aprendan a usarlas sus usuarios, preguntándose por el modo para aprender a usarlas inteligentemente, humanamente, para crear y consumir los medios digitales. Para entender su último libro, Why You Need Digital Know-How. Why We All Need It, hay que remontarse a uno anterior, Smart mobs: the next social revolution, en el que analiza el modo en el que los movimientos sociales han usado desde los años 90 las nuevas tecnologías, y utiliza el término de "inteligencia colectiva" de Pierre Levy para referirse a los modos en los que los movimientos se organizan sin líderes, con aportaciones de multitudes de personas conectadas virtualmente. De esta forma, en Multitudes inteligentes augura, desde un optimismo tecnológico, una nueva forma de comunicación y de relaciones sociales que cambiará la manera de organizarse, crear, consumir, movilizarse; una nueva forma cultural donde la interacción mediada por dispositivos tome un papel central.

En Net Smart: How to Thrive Online, Rheingold se pregunta si este saber-hacer que tienen que incorporar los individuos para organizarse a través de multitudes inteligentes en red está disponible o es posible para todos, y de qué modo la alfabetización digital (Rheingold 2012: 2) puede favorecer la distracción o bien ampliar y aumentar nuestras habilidades mentales. Lo interesante, desde esta perspectiva, es ver cómo se está formando la cibercultura y qué tipo de aprendizajes se están configurando en tanto que rasgos culturales propios de la comunidad participante. En este sentido, la alfabetización digital sería el aprendizaje de habilidades (2012: 4) que pueden favorecer el cambio y el empoderamiento de los individuos que participan en la comunidad virtual.

En la misma línea, Sergio Martínez (2011), utiliza el concepto multitudes inteligentes para aplicarlo al movimiento $15 \mathrm{M}$ en Madrid, y conocer de qué forma estas multitudes inteligentes se apropian del espacio urbano en contextos donde el tiempo y el espacio se han resignificado. Estos espacios urbanos son, con la introducción de las nuevas tecnologías, espacios híbridos en los cuales las TIC favorecen otras formas de relaciones sociales.

El objetivo de estos estudios es ver cómo se organiza la vida social a partir de la comunicación mediada por ordenador. Sin embargo, no tienen en cuenta los aspectos culturales fuera de la red. Escobar (2005) critica que, en ellos, no se tome en consideración los aspectos offline, por lo que los individuos aparecen descontextualizados, y advierte sobre los discursos desinhibidores y democratizadores de Internet que tienden a obviar los aspectos socioculturales o de género.

\subsubsection{Cibercultura con la introducción del relativismo cultural y de la interculturalidad}

Los autores recogidos en este apartado critican la concepción de cibercultura como homogénea y autónoma de las realidades sociales y culturales fuera de la red, y, por tanto, plantean que no se pueden estudiar las comunidades virtuales como encerradas en sí mismas sin tener en cuenta cómo se relacionan con otras comunidades virtuales así como la relación que mantienen éstas y, en particular, los agentes sociales con el mundo "real" y "físico" (Ardèvol 2003: 10). Por ello, si hay que tener en cuenta el contexto en que se enmarcan estas realidades, se hará también con respecto a su heterogeneidad social y cultural, e ir viendo las interrelaciones que se dan en cada caso entre la vida online y la offline, para no caer en el etnocentrismo tecnológico, es decir, para no pensar que todo agente social que navega en un chat, por ejemplo, lo hará de la misma manera. Así, se trata de dar cuenta de las vinculaciones que se establecen entre el mundo real y el virtual, más que de encontrar las diferencias entre uno y otro.

Los estudios online/offline muestran que es difícil, por no decir imposible, separar las disposiciones de clase, género, diferencias sociales y culturales cuando participamos en la red. Éste es uno de los resultados expuestos por Natalia Rybas y Radhika Gajjala (2007). Tras una investigación con jóvenes escolares de origen mexicano que residían en Ohio, concluyen que estos jóvenes reflejaban los mismos estereotipos y representaciones del yo cuando usaban las redes sociales que en la cotidianidad offline escolar. Según estas autoras, las posturas que tratan la cibercultura como un "nuevo modelo cultural" creen que con el uso de avatares los estereotipos de género o raza desaparecen en lo online, debido a la posibilidad de elegir la presentación personal, de sí mismo en la red. Sin embargo, más allá de la oposición binaria online/offline, las experiencias y prácticas aprendidas offline en los múltiples contextos socio-económicos y culturales influyen en las prácticas online, siendo imposible escapar de la identidad 
del mundo "real" por completo, al no poderse dejar de utilizar los conocimientos propios, experiencias y valores al navegar por la red. Las subjetividades de raza son producidas en contextos específicos, incorporando los procesos socioeconómicos y sociopolíticos locales y globales a través de otras categorías como las de clase, género, geografía, casta, colonización, etc. Utilizan el concepto Cybertype Framework para referirse al modo en el Internet propaga, disemina, y comercializa las imágenes de raza y el racismo respondiendo a cuestiones económicas y a las dinámicas de acceso, ya que son aquellos que pueden acceder a Internet de forma habitual los que más influyen en la reproducción de estos cibertipos. Para estas autoras, los aspectos ciberculturales no pueden entenderse sin los aspectos culturales, es decir las nuevas tecnologías no darían lugar a otras formas o modelos culturales porque, aunque se asuman otros roles, la identidad del usuario es tomada de sus experiencias tanto offline como online.

Gloria Bonder (2008), utilizando las categorías dicotómicas desarrolladas por Prensky, esto es, los nativos e inmigrantes digitales, intenta dar cuenta -a partir de una investigación sobre los programas de alfabetización digital de jóvenes latinoamericanos/as- de la construcción de la juventud como un grupo homogeneizado por las TIC, provisto de destrezas inalcanzables por la mayoría de las/os adultas/os y diferenciado de estos. La juventud encarna la revolución digital, lo nuevo, el futuro, la innovación y, en gran medida el progreso, pero estas categorías ocultan las desigualdades socioeconómicas, geográficas o de género. Bonder relativiza de esta manera la visión utópica de la cibercultura (2008: 919) a través del abordaje de dos corrientes teóricas actuales (2008: 920): por un lado, la visión deconstructivista, según la cual hay que replantearse las TIC y preguntarse si éstas no son de nuevo un tipo de exigencia globalizadora y exclusionista y, por otro lado, la visión postmodernista, que mantiene que democratizar el acceso a estos "bienes públicos" es una cuestión de derecho de ciudadanía y justicia.

\subsubsection{Cibercultura como producto cultural}

Desde esta aproximación teórica, la cultura es un conjunto de prácticas sociales que tienen que ver con la producción de conocimiento y de sentido, y lo que habría que estudiar es cómo la gente otorga significado a su experiencia, a sus emociones y pensamientos, cómo lo atribuye a las cosas, a la visión que tiene del mundo (Geertz 1987) a través de su uso de Internet. Se tiene en cuenta, por tanto, que éste no sólo está constituido por ordenadores interconectados, también por prácticas sociales significativas (Ardèvol 2003: 13). Esta propuesta teórica así, supone que la cibercultura es un producto cultural más, cuyo sentido emerge en las prácticas (Estalella y Ardèvol 2010: 2).

Christine Hine (2004), plantea una metodología de investigación sobre Internet para el estudio empírico de sus usos, a través de la cual la perspectiva etnográfica puede adaptarse a la comprensión de cómo se constituye Internet. Para Hine la metodología de una etnografía es inseparable del contexto donde se desarrolla, y sugiere una etnografía adaptativa que reflexiona alrededor del método. Por ello, el trabajo como etnógrafos comienza por dialogar con las proyecciones teóricas acerca del significado de Internet (2004: 23).

Su argumento es que existen dos maneras diferentes de concebir Internet, cada una de ellas con ventajas analíticas particulares e ideas propias acerca de lo que es un contexto de estudio adecuado. La primera sostiene que Internet representa un lugar donde se gesta una cultura: el ciberespacio. Rheingold (2004), por ejemplo, sostiene que la comunicación mediada por ordenador (CMO) es capaz por sí sola de proveer de formas de interacción y relaciones entre agentes sociales muy ricas, además de proporcionar un espacio idóneo para la conformación de comunidades. La CMO estableció la idea de Internet como cultura, proponiendo estudiar en estos espacios online los usos que las personas confieren a la tecnología, y presentando el ciberespacio como un lugar plausible para realizar trabajo de campo (Hine 2004: 19). La segunda manera de concebir Internet lo muestra como un artefacto cultural, "generada por personas concretas, con objetivos y prioridades contextualmente situados y definidos y, también, conformada por los modos en que ha sido comercializada, enseñada y utilizada"(2004: 19); por tanto, es el resultado de un proceso activo de construcción. Internet, desde este punto de vista, es un objeto o artefacto cultural como cualquier obra manual realizada con un propósito o función técnica específica: "Internet está en todas partes, pero no del mismo modo" (2004: 43), es decir, si entendemos Internet como un objeto, éste tendrá diferentes significados de acuerdo a quién lo usa y en qué contexto, de las expectativas acerca de lo qué es y para qué se puede utilizar. Por consiguiente, los significados de 
Internet adquieren socialmente su forma, siendo un producto del contexto social. Esta construcción social de la tecnología es el resultado de un proceso de negociación entre diferentes grupos de interés que ven en ella distintas ventajas y desventajas (2004: 46). Decir finalmente que una de las características de la tecnología como artefacto cultural es la flexibilidad interpretativa, ya que el significado que se le confiere viene determinado por el moldeado situacional, así que el contexto local de interpretación y uso conforma el campo de estudio etnográfico.

Según Hine, la mayoría de los estudios se han centrado en la primera concepción de Internet como cultura, omitiendo su comprensión como un artefacto cultural. Esto se debe a una dificultad para limitar el campo, es decir, a la complejidad de establecer los límites en las investigaciones con las nuevas tecnologías. Según Hine, si se quiere dar cuenta, primero, de los espacios sociales en los que Internet se emplea (offline) y, segundo, de los que emergen de su uso (online), se debe tomar en consideración dos dimensiones, a saber, la perspectiva de Internet como artefacto cultural y la perspectiva de Internet como cultura. Esto lleva a repensar la relación entre el ciberespacio y la etnografía (2004: 54). Para ella, al igual que para Rheingold, la investigación online, o la investigación etnográfica a través de la comunicación mediada por ordenador, permite reflexionar sobre lo que significa ser parte de Internet.

Díaz de Rada (2010) distingue entre etnografía en la red, que es aquella que toma la red como otra herramienta de investigación, y etnografía de la red, que se centra en el estudio del entramado complejo de instituciones presentes en la red, desde instituciones tecno-científicas a instituciones de producción, emisión y distribución de productos y contenidos, instituciones publicitarias, etc. Para este autor, ya sea en red o de la red, la etnografía sólo puede ejercerse tramando el conjunto de relaciones que afectan a las prácticas online y offline de sus agentes sociales, ya que si se investiga sólo las prácticas online se cae en el error de creer que todo sucede en o través de la red y, por tanto, de tomar por "realidad" una mera ideología. Por ello, el etnógrafo debe salir de la investigación online y contemplar las prácticas mediadas computacionalmente como un "producto de procesos comunicativos más generales" (2010: 43). De hecho, está mediada por un conjunto de técnicas y tecnologías que los seres humanos hemos desarrollado para organizar nuestra acción social, por rutinas y retóricas planificadas de forma instrumental, según sostiene Diaz de Rada (2010: 45). Por tanto, somete también a crítica el supuesto de la condición aislada de la comunicación por ordenador, puesto que desde su posición no hay comunicación humana, incluida la comunicación cara a cara, que no esté condicionada por esos artefactos institucionales históricamente configurados. Dicho esto, el autor se pregunta sobre la capacidad real que tienen estas tecnologías para configurar relaciones con y entre las personas, en las que lo virtual no se revele como lo opuesto a lo real sino, como una de sus formas institucionales.

Arturo Escobar (2005) recoge de nuevo la distinción entre una forma estrecha y una forma ancha de entender la tecnología. Desde los enfoques convencionales, la tecnología es identificada, de una forma estrecha, con herramientas. Así, la historia de la tecnología se asocia a la historia de estas herramientas y al desarrollo económico y bienestar social y humano. La teoría subyacente que se encuentra aquí es, según Escobar (2005: 17), que la tecnología y la ciencia son algo externo a la sociedad, y que las dos primeras inducen al progreso de esta última de forma automática. Según Escobar, tanto las comunidades técnico-científicas como las ciencias sociales se han comenzado a interesar por dos nuevos enfoques: el de los estudios de ciencia y tecnología, y el de los programas de ciencia, tecnología y sociedad. Estos enfoques han desplazado la anterior linealidad con que se examinaba el cambio tecnológico. En el centro de esta renovación de enfoques está el constructivismo social, y, por tanto, evidenciar que los procesos sociales son inherentes a los cambios tecnológicos.

Para ello Philip H. Howard (2002) propone el uso del concepto Network Etnography, para referirse al estudio de los usos de las nuevas tecnologías online/offline utilizando herramientas de investigación cualitativas, aunque adaptadas al campo.

El constructivismo social ha introducido en este campo de estudio conceptos innovadores como el de flexibilidad interpretativa. Ahora bien, esta perspectiva también ha sido criticada, por ejemplo, desde los planteamientos del relativismo cultural y de la interculturalidad de la cibercultura, resaltando que incide sobre los aspectos reproductivos de las tecnologías olvidando el trasfondo cultural que condiciona la interpretación y la práctica de las mismas. 
Bruno Latour introduce la perspectiva de la teoría del actor red en Reensamblar lo social (2005), obra en la plantea avanzar en un modelo que redefina lo social como el resultado de interacciones concretas y materiales entre elementos que en sí son no-sociales, criticando la ontología de la sociología tradicional. La teoría del actor red (TAR) propone el estudio de los ensamblajes de aspectos heterogéneos: la tecnología, lo económico, lo organizativo, lo político etc. Uno de los conceptos introducidos por la TAR es el de agencia para referirse a la capacidad de acción de "no humanos" (herramientas, máquinas, archivos, proyectos, edificios...). Esta agencia, se entiende contextualizada en el ensamblaje, y pierde su sentido si es sacada fuera de él y se la aísla de la totalidad (Latour 2008: 59).

En su obra "Etnografía de un caso de 'altatecnología': sobre Aramis", Latour plantea una interrogante: cómo entender la construcción social de los artefactos unida a la construcción técnica de la sociedad. La solución que propone es el uso de una nueva terminología y de herramientas metodológicas que permitan verlo como co-producción. Llama "casi objetos" a los proyectos que aún no pueden ser calificados o bien como relaciones sociales o bien como cosas. Para ejemplificar su perspectiva, acude al sistema de transporte urbano de alta tecnología, analizando el servicio de gestión de transporte conocido como programa Aramis e intentando mostrar, por un lado, cómo las tecnologías modernas son sociales, y, por otro, la construcción técnica de los lazos sociales. Para Latour, no tiene sentido establecer dicotomías entre técnicas, de una parte, y relaciones sociales, de otra. Desde su punto de vista, es un error ver a los actores sociales como meros impresores de sus intenciones o "escribidores" de sus voluntades sobre inertes objetos, como también lo es ver las tecnologías como autónomas, como imponiéndose sobre las débiles voluntades humanas. Es preciso, por el contrario, fijarse en los intercambios entre los intereses humanos traducidos y las competencias delegadas de los no humanos. Critica el constructivismo social, calificándolo de idealista y vacío cuando éste utiliza ese paradigma dualista descrito hace un momento. Según Latour, no se puede colocar el objeto en un extremo mientras se coloca en el otro lo social: "Un objeto técnico -al menos, mientras existe- es la transacción institucionalizada mediante la que se reconfiguran y traducen elementos de los intereses de los actores, al tiempo que se promueven, cambian, despliegan o combinan las competencias de los no-humanos" (Latour 1993: 95).

Para Latour, nunca existieron los objetos tecnológicos, sino que las nuevas tecnologías, al igual que las "no modernas", son asociaciones de humanos y no-humanos (Latour 2007). Así, la sociedad y la tecnología son un ensamblaje, y para él la única investigación posible es aquella que presta atención a los puntos de transacción, allí donde se efectúan las traducciones entre la sociedad y la tecnología (Estalella 2005).

\subsection{TIC y educación}

En la siguiente sección se presentan algunos de los trabajos que han analizado las TIC en espacios educativos. Antes de comenzar, hay que destacar que gran parte de ellos se han centrado en infancia o personas jóvenes en contextos escolares o en la integración de las TIC en la universidad. Aun así, es pertinente reflexionar sobre algunas de las diferentes perspectivas que se desarrollan en el ámbito de la educación y las TIC.

\subsubsection{Desde una perspectiva de los derechos de ciudadanía, el imperativo tecnológico}

Los autores aquí recogidos se refieren a la alfabetización tecnológica como un imperativo, en especial la "alfabetización digital". Se defiende la necesidad de modernizar a los agentes sociales para las nuevas sociedades postmodernas, asumiéndose como un mandato que hay que incorporar en los espacios educativos de las sociedades informacionales (Ayús Reyes 2005: 25). Desde ésta perspectiva la comunicación mediada por ordenador está configurando nuevos modelos culturales o nuevas formas de comunicación, relacionándose estrechamente con el derecho a la ciudadanía en la era digital.

De Pablos Pons et al. (2010) comparan el modo de aplicación de las políticas educativas sobre Innovación y TIC en cuatro comunidades autónomas diferentes. Según los autores, las políticas educativas emprendidas para potenciar las mismas en estos centros están incrementando el uso efectivo 
de las TIC en el currículo escolar; sin embargo, las innovaciones pedagógicas que se desarrollan con ellas no se han generalizado (2010: 3). Para ellos, la evolución de la sociedad española hacia la modernidad, la incidencia de las tecnologías o la presencia de nuevas demandas sociales, formativas y económicas hacen necesario dar cobertura a nuevas competencias para la ciudadanía, donde el sistema educativo tiene un papel importante (2010: 26). El impacto de la digitalización e Internet exigen al mundo educativo un cambio en la pedagogía para integrar estos cambios. Tras el análisis comparativo de los centros que estudian llegan a la conclusión de que los factores que intervienen en el uso innovador de las TIC por los docentes son la dotación de infraestructura, el clima de centro y los papeles de los agentes educativos, esto es, hay implicada en ello dimensiones políticas, institucionales y personales (2010: 47). Añaden que los centros educativos son entornos culturales clave para las innovaciones apoyadas en las TIC. En este estudio, sin embargo, no se reflexiona sobre los conceptos o las categorías que se manejan, la cibercultura aquí es el nuevo modelo al que hay que adaptarse, preocupándose por enculturizar a los sujetos a este nuevo modelo en lugar de hacer una análisis del mismo, de sus necesidades y beneficios.

Desde esta misma perspectiva, a saber, que el sistema educativo se tiene que adaptar al imperativo de la cibercultura como modelo cultural actual, el proyecto de investigación de Alonso Cano et al. (2010) busca, por un lado, conocer las políticas institucionales desarrolladas en Cataluña durante los últimos 20 años y, por otro, ver cómo se desarrollan en cuatro centros de enseñanza los discursos vinculados a estas políticas y las estrategias que se elaboran tanto por parte de los centros como de los profesores. Según los autores, el departamento de educación de la comunidad autónoma de Cataluña, desde la década de los ochenta, ha ido transformando su política con relación a las TIC en el ámbito de la educación, centrándose en un primer momento en la dotación de máquinas a los centros y en el uso técnico de éstas, basándose en la idea de que el ordenador constituía un recurso de "aprendizaje privilegiado" (2010: 72), pero sin plantearse transformar las practicas docentes. No es hasta el año 2007 cuando se empieza a notar un cambio en el discurso de las administraciones públicas catalanas, pasándose de hablar de TIC a TAC (tecnologías para el aprendizaje y el conocimiento); cambio terminológico a través del cual se pretende poner al mismo nivel de importancia la parte técnica y la parte pedagógica (2010: 59). No obstante, este cambio del discurso desde las TIC a las TAC se ha originado lejos de la realidad práctica diaria de los centros, por ello, los autores animan a que las administraciones públicas consideren el sistema educativo como un todo, integrando y coordinando la puesta en práctica de los decretos, instrucciones y recursos en los centros educativos. Valverde (2010) llega a conclusiones parecidas, exponiendo que a pesar del discurso político que subraya el potencial de las TIC, no han sido implementadas en las aulas con todas sus consecuencias. Aunque la aplicación integrada de las políticas educativas de las TIC ha mejorado las infraestructuras y el equipamiento en los centros educativos, estas políticas no han producido los cambios significativos anunciados. Los profesores según el autor- no están completamente preparados para integrar las TIC en el aula, y están utilizando sólo las posibilidades básicas de éstas como, por ejemplo, la recuperación de la información.

Consideradas bajo la perspectiva de que las TIC están transformando nuestra vida personal y profesional, Escontrela y Stojanovic (2004) defienden también que hay que cambiar las estrategias y objetivos pedagógicos en las escuelas si queremos que los futuros estudiantes tengan las habilidades que la nueva sociedad informacional les exige, que la "enseñanza [esté] centrada en el alumno" o "aprender a aprender" (2004: 2). Como De Pablos Pons, estiman que para la introducción efectiva de las TIC en el aula es necesario un enfoque que integre las políticas educativas, la organización de la institución, los recursos materiales y los actores involucrados. Para llegar a esta conclusión se apoyan en una investigación realizada por IN3-UOC (2004) sobre la incorporación de Internet en la educación primaria y secundaria de Cataluña y su relación con la organización, la cultura y las prácticas educativas de los centros docentes. Se interesan por conocer cómo los responsables de los centros, los profesores y los alumnos utilizaban ( $\mathrm{y}$ con qué finalidad) Internet en las diferentes actividades de la cotidianidad de los centros, y en qué medida ello contribuye a la aparición de una nueva cultura educativa.

Estos análisis platean, al igual que Carles Monereo (2005), que gracias a las TIC se ha iniciado un nuevo proceso de socialización y culturalización (2005: 8), y que el cambio tecnológico está propiciando nuevas formas de aprender, de buscar información, de comunicarnos, que pasa por la adquisición de unas competencias básicas. Es decir, el nacimiento de un nuevo modelo cultural al que hay que adaptarse e 
introducir en los centros educativos. Un modelo cultural que implicaría un nuevo escenario pedagógico en el que las metodologías tradicionales quedan obsoletas. Garrison y Anderson (2005), citando a Brown (2000), aseguran que el e-learning, por ejemplo, todavía está por consolidarse como modelo, con características sin explotar (Garrison y Anderson 2005: 17). Para ellos, esta nueva "ecología educativa" basada en una forma diferente de comunicación no puede simular el sistema presencial tradicional. Para estos autores, e-learning se basa en una "comunidad de investigación" (2005: 21), en la que los avances tecnológicos hacen que los profesores se replanteen la manera de enfocar la enseñanza, suponiendo nuevas oportunidades de conocimiento intercultural (2005: 22). Garrison y Anderson consideran que es simplista tratar las nuevas tecnologías como meros transmisores de información, asegurando que dan una nueva forma a las experiencias y a la cosmovisión del mundo (2005: 24). Otra de las metodologías pedagógicas es la planteada por Lourdes Morán (2012), el blended learning o b-learning, combinación de educación a distancia y presencial (2012: 2). Esta propuesta responde a la concepción de una nueva forma cultural que trata de desentrañar y potenciar los nuevos lenguajes y formas de interacción. Según la autora, ante la radical transformación de la cultura de los jóvenes es imprescindible una nueva forma de relación entre los profesores, los alumnos y los contenidos (2012: 2) que tengan en cuenta las nuevas tecnologías.

Para finalizar este apartado, se puede destacar que, en los diferentes estudios que se han desarrollado sobre las nuevas tecnologías en espacios educativos desde la perspectiva del imperativo tecnológico, la adquisición de las competencias o alfabetización digital no es algo que se cuestione, sino que lo que se cuestiona es por qué muchas de las políticas públicas no están funcionando. La mayoría de esos estudios llegan a la conclusión de que, además del acceso a las nuevas tecnologías, también es preciso incorporar nuevas pedagogías o modelos educativos que conlleven nuevas formas de enseñar, esto es, un modelo cultural en el que las nuevas tecnologías tengan un papel central para que profesores y alumnos respondan a las exigencias de la sociedad informacional, para la inclusión efectiva cibercultural. Para que estas pedagogías funcionen tienen además que integrarse y coordinarse todos los agentes educativos involucrados en el proceso educativo. De este modo, como otros muchos trabajos que tratan sobre las nuevas tecnologías, los abordados en este apartado no dejan de tener, si no un carácter profético, sí prescriptivo, que a veces predomina sobre su intención descriptiva o analítica.

\subsubsection{La alfabetización desde una perspectiva crítica}

Hay que tener en cuenta que las posturas desarrolladas a continuación no presentan una crítica al imperativo de la Sociedad Informacional, sino a las desigualdades que el propio sistema está creando, tales como la división o brecha digital o la alfabetización tecnológica o digital, ambos conceptos discutidos en los siguientes textos.

Begoña Gros y David Contreras (2006), encuentran este imperativo tecnológico central para la formación de la ciudadanía del siglo XXI, en la que la tecnología es una herramienta indispensable para la participación socio-política y económica (2006: 103). Para ellos, la sociedad informacional ha beneficiado únicamente a unos pocos, aumentando la brecha ya existente entre países y grupos sociales, también llamada la "brecha" o "división digital" (2006: 106), que excluye a personas y grupos sin acceso ni a las tecnologías ni al conocimiento requerido para su manejo. Para estos autores, es importante la formación de las "nuevas generaciones" (2006: 107) en nuevas formas de alfabetización, la alfabetización digital, para que no queden al margen del mundo digital, para el desarrollo de competencias ciudadanas en la sociedad informacional.

Para alcanzar esta alfabetización digital, Gros y Contreras proponen una metodología que escape de la pedagogía tradicional de retención de contenidos por parte del aprendiz. Así, se trata de la creación de entornos de aprendizaje que permitan presentar al estudiante variadas experiencias y escenarios para la construcción del conocimiento por sí mismo. Los autores lo llaman "aprendizaje experiencial y participativo" (2006: 110). Citando a Wenger (2001), definen el aprendizaje como "un proceso interactivo de participación en las prácticas culturales y en las actividades compartidas por las comunidades sociales" (Gros y Contreras 2006: 112), y lo entienden un proceso de incorporación creciente del aprendiz como miembro de una comunidad (Lave y Wenger 1991). Las TIC toman relevancia en este proceso ya que pueden contribuir a la descentralización de las formas de aprendizaje y a la construcción colectiva del conocimiento, formando redes de discursos, un medio de búsqueda, expresión, 
Frente a estos autores que hablan principalmente de alfabetización digital, Bautista García-Vera (2007) se preocupa por cómo integrar en el contexto educativo lo que él considera las tres alfabetizaciones más importantes: la textual, la audiovisual y la digital, y propone como solución la alfabetización tecnológica multimodal o múltiple. Sostiene, citando a Freire (2007: 591), que al igual que la alfabetización textual es tomar distancia de los acontecimientos para analizarlos y representarlos en el texto, la alfabetización audiovisual no tiene que buscar sólo el aprendizaje del análisis de los mensajes sonoros e icónicos, sino además la producción audiovisual que permita participar en el entorno social y cultural (2007: 592).

Según Bautista, en el siglo XX el desarrollo tecnológico propició que haya productos donde confluyen los sistemas de representaciones tanto textuales como audiovisuales. Pero de nuevo es necesaria la alfabetización para una mirada crítica y la producción creativa de mensajes e informaciones a través de los medios digitales, si no queremos que la brecha tecnológica entre pueblos y capas sociales de la población aumente. Por tanto, la disminución de la brecha tecnológica tiene dos dimensiones, una dimensión material relativa al acceso al dispositivo o a la red; y una dimensión funcional, el uso que se hace de éstos (2007: 593). La alfabetización tecnológica es multimodal porque tiene que recoger el aprendizaje de los diferentes lenguajes textuales y audiovisuales, así como los medios para el análisis y producción a través de las herramientas digitales. El autor añade que las nuevas tecnologías posibilitan tanto el uso y elección de múltiples lenguajes como la comunicación distante entre diferentes personas con distintos mensajes, lo que permite más caminos y formas de comunicación multiculturales. Por ello, Bautista asocia la interculturalidad a la "alfabetización tecnológica multimodal" (2007: 595), y asegura que ésta es una manera de responder al reto educativo de encontrar modos de participación e independencia de los participantes en el proceso educativo.

Bautista habla de brecha tecnológica en lugar de brecha digital, afirmando que la desigualdad no se refiere sólo a no poder acceder a los canales de comunicación, sino que con ello "se evita el desarrollo de saberes y estrategias sociales implícitas en las relaciones humanas" (2009: 50) y, consecuentemente, la incorporación al mundo social y laboral que exige la sociedad informacional. Por tanto, la disminución de la brecha tecnológica pasa por la creación y narración de historias en el seno de los diferentes grupos utilizando las tecnologías audiovisuales por parte de sus miembros. Además, la alfabetización tecnológica también pasa, según este autor, por integrar las competencias y conocimientos vinculados al mundo de la informática (2009: 48), para ello propone proporcionar formación a los alumnos en nuevas tecnologías a lo largo de la escolaridad obligatoria.

Para Area Moreira (2002), la introducción de las nuevas tecnologías en la cotidianidad es un hecho imparable que reporta beneficios económicos, sociales y culturales para aquellos que las usan, aunque existen efectos secundarios, como es aumentar el distanciamiento económico y cultural entre aquellas personas que tienen acceso y aquellas que no. Este distanciamiento está creando un tipo nuevo de analfabetismo, que consiste en la incapacidad para acceder a la cultura vehiculada a través de las TIC. Por tanto, para Area Moreira, las políticas sociales y educativas que ayuden a disminuir este distanciamiento son imprescindibles si se quiere una sociedad informacional para todos. Hoy en día, sigue afirmando este autor, la comunicación y el conocimiento no sólo se transmiten a través del lenguaje escrito, sino también a través de otros lenguajes como el audiovisual. Así, el concepto de alfabetización cambia radicalmente, pudiéndose hablar de analfabetización tecnológica. Esto quiere decir que aquellas personas que no estén cualificadas para el uso de las TIC estarán marginadas culturalmente y del mercado en las sociedades informacionales.

La alfabetización tecnológica, como proyecto democrático y participativo de los ciudadanos, requiere aprendizaje instrumental y cognitivo de las mismas, pero además "plantear y desarrollar valores y actitudes de naturaleza social y política" (2002: 6). Para poder democratizar la alfabetización en todas sus dimensiones, propone alternativas de naturaleza política que compensen las desigualdades educativas en este ámbito tanto en el sistema escolar como en la formación ocupacional y la educación no formal.

Santoveña (2011) apuesta por el aprendizaje colaborativo, siendo éste "una forma de organización social del aula y de los procesos de enseñanza y aprendizaje basada en la interdependencia positiva de 
objetivos y recursos entre los participantes" (2011: 4). La alfabetización la entiende a partir de un concepto que desarrollan Gee, Lankshear y Knobe (2011: 5), quienes sostienen que cuando se juega con un videojuego, por ejemplo, se está embarcando en una nueva alfabetización, de una parte porque se adquiere un lenguaje, pero también porque se aprende a leer gráficos, símbolos, imágenes, etc. Así, hay una multiplicidad de alfabetismos, es decir, cada vez que un sujeto se enfrenta a un nuevo medio, a un nuevo formato, a un nuevo impreso, se ponen a su disposición diferentes tipos de descodificaciones. Santoveña plantea que lo "nuevo" es un fenómeno social y, por tanto, los nuevos alfabetismos sólo pueden investigarse desde un punto de vista socio-cultural, concebidos como tipos de prácticas que implican una nueva forma de pensar, con especial importancia de la colaboración, la participación y la dispersión (2011: 5).

Brown (2005), añade que con el desarrollo de las nuevas tecnologías en la educación (2005: 4), se ha pasado de la "producción del conocimiento" a la "configuración" del mismo, lo que significa que las instituciones educativas tienen que desarrollar las competencias necesarias en sus aprendices para gestionar y configurar el conocimiento de manera efectiva, de manera que el profesor pasa de "enseñar" a "facilitar el aprendizaje" (2005: 4). Basándose en Soloway, predice que los nuevos enfoques educativos -utilizando las TIC- buscan facilitar y apoyar la investigación del aprendiz, centrando su atención en las cuestiones generadas desde el estudiante. Para él, el futuro de la educación pasa por dejar de proveer contenidos al aprendiz y tratar, por el contrario, de fomentar la mejora de sus habilidades para que encuentre, modifique, evalúe y manipule la información y el conocimiento, y así lo pueda integrar en el mundo del trabajo y en la vida cotidiana tanto para solventar problemas como para comunicar este conocimiento a otros (2005: 10).

Para resumir: se relativiza el concepto de cibercultura, y se toma en consideración el análisis de ésta como artefacto cultural en el que tener en cuenta la diversidad y heterogeneidad de prácticas de los agentes sociales, coincidiendo en que no basta con poner a disposición de las personas los dispositivos tecnológicos, sino que es preciso desarrollar una serie de pedagogías que promuevan la capacitación de habilidades para utilizar esos dispositivos. Entienden que la sociedad informacional es el presente y que la participación ciudadana en el ciberespacio pasa por aprender a "navegar" y comprender los nuevos campos de acción.

\section{Conclusiones}

Mientras que los trabajos que se mantienen dentro de la idea del imperativo tecnológico están más cercanos al determinismo tecnológico y enfocan la cibercultura como nuevos modelos o formas culturales, los estudios más críticos entienden las nuevas tecnologías como artefactos culturales. Desde la construcción social de la educación y de las nuevas tecnologías es posible, como se ha visto, repensar los procesos educativos teniendo en cuenta a los agentes sociales y los múltiples discursos y formas de comunicación. Sin embargo, la Teoría del Actor Red ha criticado esta postura constructivista porque sigue dicotomizando entre nuevas tecnologías y viejas tecnologías, entre viejas y nuevas formas de enseñar, etc. Por ello, se anima a producir investigaciones que cuestionen estas categorías y ayuden a comprender los usos que las personas hacen de las nuevas tecnologías.

Aunque hay estudios relacionados con las nuevas tecnologías en los espacios educativos, estos se han centrado en analizar la situación de los centros con la introducción de dispositivos tecnológicos, en prescribir mejoras para la integración de estas tecnologías en los contextos educativos o proponer pedagogías con el objetivo de que las nuevas necesidades de ciudadanía en la era digital lleguen al máximo número de personas, y en el mejor de los casos, tienen en cuenta las peculiaridades culturales, sociales y económicas. No en vano, la mayor parte de estos estudios se han llevado a cabo gracias a la financiación de instituciones que se interesan por conocer el estado de los centros y adaptar sus administraciones educativas a las nuevas "imposiciones" de la era digital, tanto material como legislativamente.

Sin embargo, ¿qué está ocurriendo en las aulas? Como se ha visto, algunos estudios subrayan que no se están aprovechando en el aula todas las posibilidades que las nuevas tecnologías ofrecen, 
limitándose a utilizarlas de forma básica. Obviando, por una parte, que no se cuestione la construcción de estas posibilidades básicas o que el sistema educativo se entienda en términos de productividad y las nuevas tecnologías como herramientas para, otra pregunta entra en consideración: ¿De qué modo afectará este tipo de aprendizaje a los agentes sociales?

La concepción de la cibercultura como un modelo cultural o una forma cultural, aunque enriquezca el análisis en ciertos aspectos como se ha visto, deja entre paréntesis al agente social y los usos situados que éste hace de los dispositivos tecnológicos, prediciendo que la introducción de las tecnologías digitales y nuevas metodologías en el aula implicaría la incorporación paulatina y beneficiosa de la cibercultura como nuevo modelo cultural.

El prolífico campo de los estudios de la cibercultura, puede aportar críticas constructivas a partir de las cuales aprehender la introducción de los dispositivos tecnológicos en los procesos educativos. Sería enriquecedor para estos estudios en y de los espacios educativos que se abordara la diversidad de formas de hacer de los agentes sociales y comprender la relación que se establece entre humanos y artefactos no-humanos, en los que las personas que forman parte del sistema educativo fueran las protagonistas y no el llamado imperativo tecnológico al que estas personas, centros y políticas se tienen que adaptar. Del mismo modo, para comprender la construcción de las nuevas tecnologías existen otros aspectos que tienen que tenerse en cuenta, como lo social y cultural, que ayudará a ensamblarlas y situarlas en el complejo entramado técnico-social, y socio-técnico. Así cabría preguntarse: ¿qué usos se está dando, por los diferentes actores, de los dispositivos tecnológicos dentro y fuera del espacio educativo? o ¿qué tipo de relaciones se están configurando en el entramado social y técnico?, y ¿de qué modo se relacionan?

Si por el contrario se sigue enfatizando cómo adaptarnos, las consecuencias interpretativas pueden confundir una necesidad imaginada con los objetivos de las investigaciones futuras.

\section{Bibliografía}

Alonso Cano, Cristina (y otros)

2010 "De las propuestas de la Administración a las prácticas del aula", Revista de Educación, n 352: 53-76.

Ardèvol, Elisenda

2003 "La cibercultura: un mapa de viaje; aproximaciones teóricas para un análisis cultural de Internet", Aportaciones al Seminario Pensar la Cibercultura, Antropología y Filosofía del Nuevo Mundo (Digital). Fundación Duques de Soria.

http://eardevol.files.wordpress.com/2008/10/eardevol_cibercultura.pdf

Area Moreira, Manuel

2002 "Igualdad de oportunidades y nuevas tecnologías: Un modelo educativo para la alfabetización tecnológica", Educar, n² 29: 55-65.

Ayús Reyes, Ramfis

2005 "Educación y nuevas tecnologías de la información y las telecomunicaciones", Perspectivas docentes, $\mathrm{n}^{0}$ 29: 19-28.

Bautista García-Vera, Antonio

2007 "Alfabetización tecnológica multimodal e intercultural”, Revista de Educación, n 343: 209-210.

2009 "La sociedad de la información versus la escuela analógica, o lo necesario de las narraciones audiovisuales", Educatio Siglo XXI, n 2: 43-52. 
2008 "Juventud, género \& tic: imaginarios en la construcción de la sociedad de la información en América latina”, ARBOR. Ciencia, Pensamiento y Cultura, nº 733: 917-934.

Brown, John S.

2000 "Growing up digital: How the Web changes work, education, and the ways people learn", Change, marzo/abril: 11-20.

Brown, Tom H.

2005 "Beyond constructivism: Exploring future learning paradigms", Education Today, n 2.

http://www.bucks.edu/old_docs/academics/facultywebresources/Beyond_constructivism.pdf

Castells, Manuel

2004 La era de la información: economía, sociedad y cultura. Madrid, Siglo XXI.

2006 La sociedad red: una visión global. Madrid, Alianza Editorial.

De Kerckhove, Derrick

1999 La piel de la cultura. Investigando la nueva realidad electrónica. Barcelona, Gedisa.

De Pablos Pons, Juan

2008 "Algunas reflexiones sobre las tecnologías digitales y su impacto social y educativo", Quaderns digitals: Revista de Nuevas Tecnologías y Sociedad, $\mathrm{n}^{0} 51$.

http://dialnet.unirioja.es/servlet/articulo?codigo $=2566547$

De Pablos Pons, Juan (y otros)

2010 "Factores facilitadores de la innovación con TIC en los centros escolares: un análisis comparativo entre diferentes políticas educativas autonómicas", Revista de Educación, nº 352: 23-51.

Diaz de Rada, Ángel

2010 "La lógica de la investigación etnográfica y la mediación computacional de la comunicación", Revista Chilena de Antropología Visual, nº 15: 40-57.

Domínguez Figaredo, Daniel (y otros)

2007 "Etnografía virtual", Forum: Qualitative Social Research, vol. 8, nº 3.

http://www.qualitative-research.net/index.php/fqs/article/view/274/603

\section{Escobar, Arturo}

2005 "Bienvenidos a Cyberia. Notas para una Antropología de la Cibercultura", Revista de estudios sociales, $\mathrm{n}^{\circ}$ 22: 15-35.

Escontrela, Ramón (y Lily Stojanovic)

2004 "La integración de las TIC en la educación: Apuntes para un modelo pedagógico pertinente", Revista de Pedagogía, vol. 25, nº 74.

http://www.scielo.org.ve/scielo.php?pid=s0798-97922004000300006\&script=sci_arttext

\section{Estalella, Adolfo}

2005 "Filtrado colaborativo: la dimensión sociotécnica de una comunidad virtual”, UOC Papers, n 1.

http://www.uoc.edu/uocpapers/1/dt/esp/estalella.pdf

Estalella, Adolfo (y Elisenda Ardèvol)

2010 "Internet: instrumento de investigación y campo de estudio para la antropología visual", Revista Chilena de Antropología Visual, nº 15: 1-21.

Garrison, D. Randy (y Terry Anderson)

2005 El e-learning en el siglo XXI: Investigación y Práctica. Barcelona, Octaedro.

Geertz, Clifford

1987 La interpretación de las culturas. Barcelona, Gedisa. 
Gros, Begoña (y David Contreras)

2006 "La alfabetización digital y el desarrollo de competencias ciudadanas", Revista iberoamericana de educación, n 42: 103-125.

Hine, Christine

2004 Etnografía virtual. Barcelona, Editorial UOC.

Howard, Philip. N.

2002 "Network ethnography and the hypermedia organization: new media, new organizations, new methods", New media \& society, $\mathrm{n}^{\circ} 4$ : 550-574.

IN3-UOC.

2004 La escuela en la sociedad red: Internet en el ámbito no universitario. Barcelona, Universitat Oberta de Catalunya.

Latour, Bruno

1993-1994 "Etnografía de un caso de 'alta tecnología': sobre Aramis", Política y sociedad, n 14/15: 7798.

2005 Reassembling the Social. Oxford, Univerty Press.

2007 Nunca fuimos modernos. Ensayo de antropología simétrica. Buenos Aires, Siglo XXI Editores.

2008 "Llamada a revisión de la modernidad. Aproximaciones antropológicas", en Sánchez Criado, T. (ed.), Tecnogénesis: La construcción técnica de las ecologías humanas. Madrid, AIBR, Antropólogos Iberoamericanos en Red: 169-195.

Lave, Jean (y Etienne Wenger)

1991 Aprendizaje situado. Participación periférica legítima. Nueva york, Cambridge University Press.

Lévy, Pierre

1999a ¿Qué es lo virtual? Barcelona, Paidós.

1999b Collective intelligence: mankind's emerging world in cyberspace. Cambridge, MA, Perseus.

2001 Cyberculture. Minneapolis, University of Minnesota Press.

Monereo i Fon, Carles

2005 Internet y competencias básicas: aprender a colaborar, a comunicarse, a participar, a aprender.

Barcelona, Grao.

Morán, Lourdes

2012 marzo "Blended-Learning. Desafío y oportunidad para la educación actual", EDUTEC, Revista Electrónica de Tecnología Educativa, nº 39.

http://edutec.rediris.es/Revelec2/Revelec39/blended_learning_desafio_oportunidad_educacion_actual.HTML

Negroponte, Nicholas

1995 El mundo digital. Barcelona, Ediciones B.

Piscitelli, Alejandro

2002 Ciberculturas 2.0: en la era de las máquinas inteligentes. Buenos Aires, Paidós.

Piscitelli, Alejandro

2006 "Nativos e inmigrantes digitales. ¿Brecha generacional, brecha cognitiva, o las dos juntas y más aún?", Revista Mexicana de Investigación Educativa, 11(028): 179-185.

Prensky, Marc

2001a "Digital Natives, Digital Immigrants", NCB University Press, 9(5).

2001b “Do They Really Think Differently?", NCB University Press, 9(6).

Prensky, Marc

2004 "The death of command and control?", Technology Alliance Partners.

http://www.marcprensky.com/writing/Prensky-SNS-01-20-04.pdf 
Rheingold, Howard

2003 Smart Mobs: The Next Social Revolution. Cambridge, Basic Books.

2004 Multitudes inteligentes: la próxima revolución social. Barcelona, Gedisa.

2012 Net Smart: How to Thrive Online. Cambridge, MIT Press.

Rybas, Natalia (y Radhika Gajjala)

2007 "Developing Cyberethnographic Research Methods for Understanding Digitally Mediated Identities", Forum: Qualitative Social Research, nº 8 (3).

Santoveña, Sonia M.

2011 "Análisis socioeducativo del blogueo como actividad alfabetizadora y colaborativa", EDUTEC, Revista Electrónica de Tecnología Educativa, nº 35.

http://edutec.rediris.es/Revelec2/Revelec35/pdf/Edutec-e n35_Santovena.pdf

Valverde, Jesús (y otros)

2010 "Políticas educativas para la integración de las TIC en Extremadura y sus efectos sobre la innovación didáctica y el proceso enseñanza-aprendizaje: la percepción del profesorado", Revista de Educación, nº 352: 99-124.

Wenger, Etienne

2001 Comunidades de práctica. Aprendizaje, significado e identidad. Barcelona, Paidós. 